\title{
Comparative Analysis of loT based Healthcare Architectures
}

\author{
Pooja M. \\ Department of Computer Science \\ Christ University, \\ Hosur Road \\ Bangalore 560029, \\ Karnataka, India
}

\author{
Deepthi Das \\ Department of Computer Science \\ Christ University, Hosur \\ Road Bangalore 560029, \\ Karnataka, India
}

\begin{abstract}
In the rising technology, the Internet of Things (IoT) comprised of physical objects that are inserted with sensors, actuators for automatic monitoring and tracking of patient's records and biomedical devices. This paper explores in detail for IoT network architectures and tells the key technologies and process of decision-making system is presented based on the understanding of parameters in each architecture. The paper focuses on the comparative analysis of smart healthcare architecture and based on a study of each architecture, the paper suggests which architecture is best to implement in the Smart Hospitals.
\end{abstract}

\section{Keywords}

Radio Frequency Identification (RFID), Internet of Things (IoT), Wireless Sensor Network (WSN)

\section{INTRODUCTION}

The Internet of Things (IoT) is an active system network structure, associating physical devices and virtual things together. It deals with the standard protocols and self-organize network and each object communicates with real world entities. The IoT applications can be used in several domains such as retail, logistics, healthcare, home automation, safety, and security monitoring.

Nowadays, the main problem faced by Healthcare systems is accessibility and affordability of quality health care services and also a lack of transparency. The idea of Internet of Things (IOT) carries an open door for communicating with a device without human intervention. The key function enabling in an IOT is smart objects, which are categorized into four characteristics, for example, identification, area, detecting, and availability. The main rising technologies for smart objects are Radio Frequency Identification (RFID), Global Positioning System (GPS), Sensor Networks and further improvements in remote availability [1]. The goal of the IOTHealthcare system is to reduce the man work, improving patient's safety and improving the quality of care. During an emergency case, the patient's suffering from chronic diseases [2] -diabetes, blood pressure, glucose, etc. will not be able to go to hospital frequently in that scenario the IOT will be helpful. WBAN (Wireless Body Area Network) is connected to the patient's body, the different sensors have been placed inside the patient. Each sensor will function according to the disease and report the data to the caregivers. The system can provide the user effective assistance about his daily self-care and in taking of medicines will improve the patients' health. If there is any necessary help the user can communicate with the care givers. Moeen Hassanalieragh, ISLAM1 and, et.al in their study of smart healthcare systems, the research come across the most important architectures which is explained in detail of the parameters, technologies and study about all the system requirements, the paper suggests which is the best architectures to implement in the hospitals.

\section{METHODOLOGY}

\subsection{Zigbee}

Zigbee, as Bluetooth, has a huge introduced base of operation, in spite of more in traditional and industrial settings. Zigbee depends on the IEEE802.15.4 protocol, which is an industry standard remote systems administration operating at $2.4 \mathrm{GHZ}$ focusing on applications that require generally occasional information trades at low information rates over a confined area and inside a $100 \mathrm{~m}$ range such in a home or building[14].

\subsection{LowPAN}

6LowPAN is a key IP (Internet Protocol) based technology is 6LoWPAN (IPV6 Low power wireless Personal Area Network). Instead of being an IoT application protocols technology like Bluetooth or Zigbee, 6LoWPAN is a network protocol that defines encapsulation and header compression mechanism [15].

\section{RELATED WORK}

IoT permits the correspondence between all the things we see around us separated from the human-machine, the IoT healthcare stages have different signals such as Electrocardiogram (ECG), Body temperature, Blood Pressure, Glucose and sensed data are transferred over a wireless network. This information are monitored by various caregivers such as medical doctors. In view of this data, patient's wellbeing conditions are naturally observed and proper inputs are given by the doctors. Many research endeavors that are concentrating on IoT structures in healthcare environments frequently utilize diverse technologies, for example, ZigBee, Wi-Fi, and RFID innovations [3], For instance, E.Serafim, and S.Motoyama [4] proposes a system model to screen patient's health through an RFID situated in a healthcare environment. In other effort Sermakani [5], discusses the general idea with respect to sensors. Islami1, et.al [6] describes how the sensors data are collected and reached the doctor with respect to video observing interface called Health Face for therapeutic healthcare systems based on the wireless body area sensor network. Health Face is known as telemedicine which can be accessed to all around, at any time via IP address.

In view of 6LOWPAN there exist a few works exhibiting architectures based on system models. For example, Lee [7] proposes structure to support network-based mobility of sensor devices for mobile healthcare. Whereas, they just 
concentrate on mobility of network without considering the constant necessities for health information in healthcare environments and nodes' battery life time. A large portion of the researcher's introduced this platform has limitations such as sensors data are collected via a gateway and send to one common doctor which leads to the problem because all the doctors are not specialized at every disease and the purpose of this paper is to analyze a current Smart Healthcare System (SHS) architectures based on customized healthcare environment, thus the research gives a comparative study of few important architectures. From this, the study of research can conclude saying that which architecture would be efficient for implementing in hospital systems.

\section{EXISTING METHODOLOGY}

Existing architecture which is considered for analysis is described in this section.

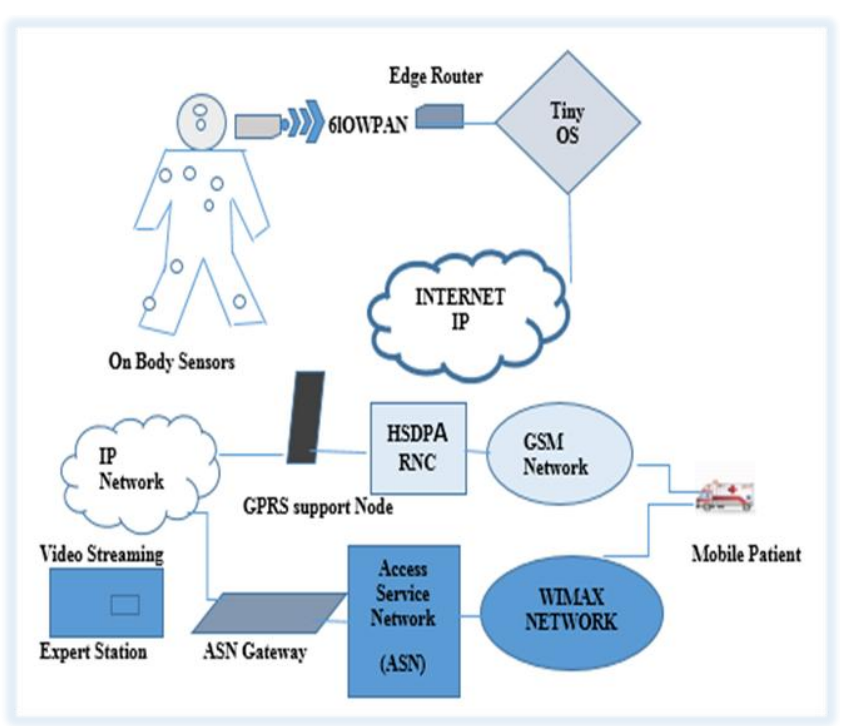

Fig 1: Remote monitoring in wearable's and Personalized health care

In Fig 1: the biographer describes the complete healthcare framework and figures a scenario in which patient's health records and vital signs (BP, ECG, Diabetes, and Heart Rate) are secured by portable medical devices and sensors attached to the patient's body. The data which are secured are stored and analyzed for further process, stored data are taken from different sensors such as BP sensors, Diabetes sensors, ECG sensors, etc. Furthermore machines gets to be distinctly helpful for accumulation. Depending on study and aggregation caregivers can screen patients from any location and react according to their need. The patients can also communicate through a video streaming by online during an emergency case as well as backings the gushing of interconnected system with overall interoperability for a microwave access (WiMAX), an internet protocol (IP) network and global system for a mobile (GSM) network as well as usual gateways and the access service networks [6]

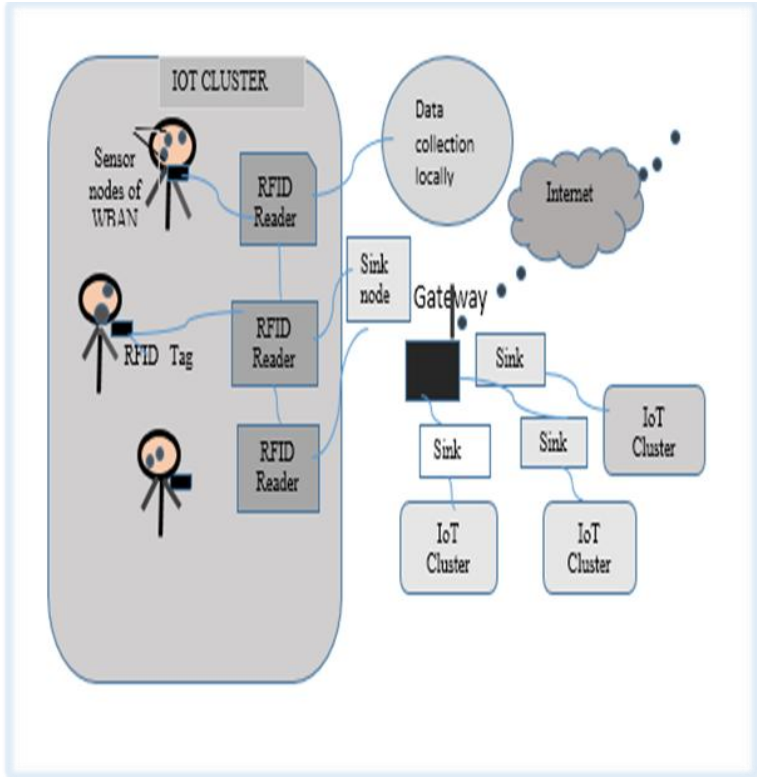

Fig 2: Network model for patient monitoring using IoT cluster

In Fig: 2, the model focuses about the vital signs of parameters which is functioning with the help of WBAN and RFIDs. The patient' body are attached with sensors through a WBAN network, the patients wearing WBAN gets a dynamic RFID tag with high range. This Active RFID tags can only read the nearest information which are constrained with range from 5 to 200 meters. The RFID tags convey data about the patient's additionally serve as the interface amongst WBAN and IOT cluster. The data is sent to a medical center or a bigger clinical which will collect the patients data, process and store them. The specialist can collect information locally from RFID readers. This can be done only when the specialist visiting a community or group. The author come across few limitations about the architecture which tells RFID tags have the capacity with limited range, beyond the range gives a network problem. The system cannot ensure the service provided for 24 hrs there would be a server problem [4]

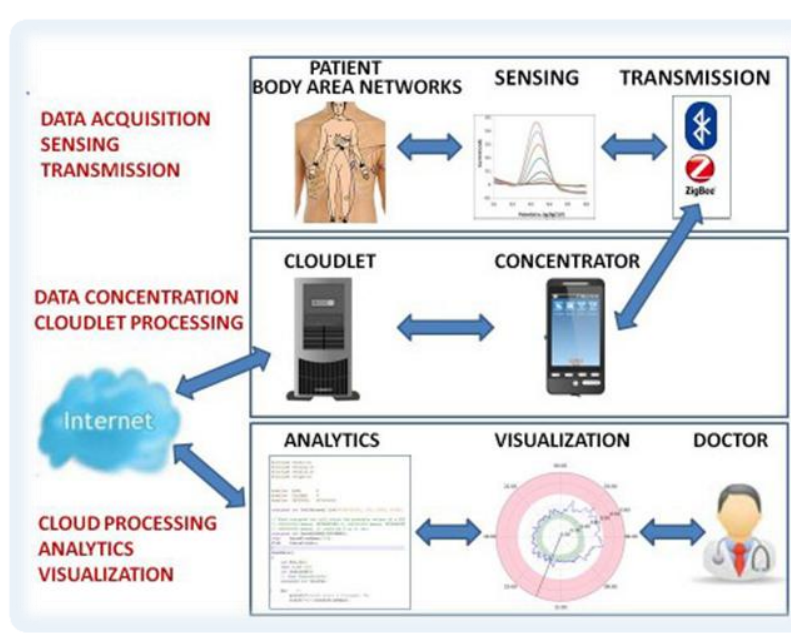

Fig 3: Remote patient monitoring based on IoT-cloud architecture

In Fig: 3, the architecture describes for a remote health monitoring system. It explains about the major components introduced in the system architecture: 
The greater part of the architecture for remote health proposal has three fundamental segments: A Wireless Body Area Network (WBAN) comprising of wearable sensors as a result of the information securing unit, correspondence and organizing and the communication and networking and the service layer. Above proposition demonstrates the framework that utilizations wearable sensors to record biomedical parameters like blood pressure and body temperature. The information are captured through an entrée server and transmit via Bluetooth. The entree server receives data and converts into concurred format and stores it in cloud for later analysis by the clinicians.

The technology visualizes a remote health systems and for information gathering, storage and access each and every records are observed for analysis.

Exact determinations and perceptions of patient's medical condition depends relies on upon investigation of medical records containing changed physiological attributes over an extended measure of time. Managing each amount of time and knowledge analysis of high spatiality makes assignment very disappointing and error prone for clinicians. In spite of the fact that the use of knowledge mining and perception methods had prior addressed as an answer to the same challenge, these procedure have only recently gained attention in remote health observation frameworks. While the presence of electronic remote health observation systems are secured to change the standard human health care services, combination of IoT healthcare frameworks they can build the intelligence, adaptability and flexibility.

Data Acquisition is executed by several wearable sensors that measures key parameters, for example, skin temperature, respiratory rate, ECG and gait (posture). The sensors associated with the system relates a transitional data, for example collector or concentrator, that is typically an advanced mobile phone arranged inside the neighborhood of the patient.

The Data Transmission parts of the framework are responsible for movement of recordings of the patient from the patient's home (or any remote location) to the info center of the Healthcare Organization (HCO) with guaranteed security and protection. The vast majority of tangible securing stage is provided with a brief vary radio, for example, Zigbee or low-power blue tooth, that utilizes to exchange sensor information to the concentrator. The HCO is more relayed on future storage victimization on the concentrator through a smartphone's WLAN or cellular learning affiliation. Based on the System design sensors within the knowledge of an Internet of Things (IoT) as every individual sensor's knowledge will be accessed through the web via the concentrator.

Cloud based data storage is used in capturing patient's medical information. Since huge volume of data are gathered in cloud it would be difficult for doctors to take a decision, therefore analytics and visualization techniques are used to make data in digestible format [8].

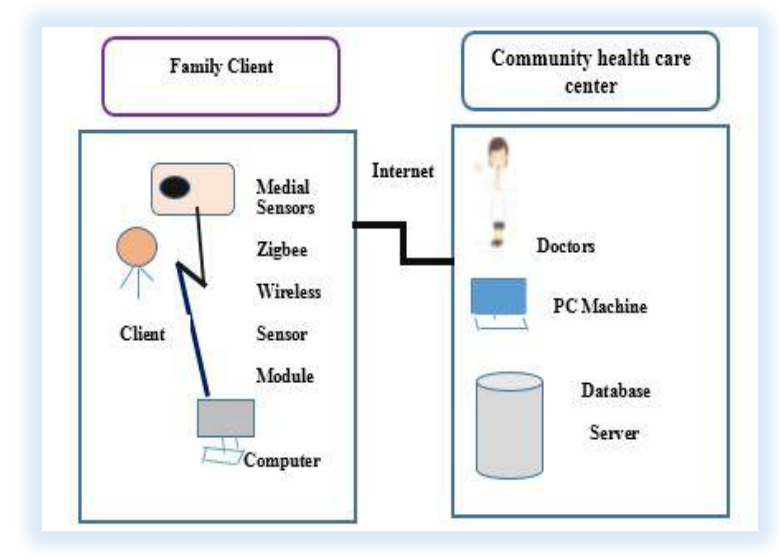

Fig 4: IoT Healthcare Architecture

The family IoT architecture [9] incorporates the medical sensors, Zigbee wireless network, and Home Computer for screening the physiological attributes to the patient's continuously. The sensors data are then transmitted to the base stations/checking stations through Zigbee remote system. Information is exchanged from the base station to the PC and information is exchanged to the health care center via Internet. The doctors are communicated via Health Care center through PC machines database servers, and medical works. The personal information, monitoring data and doctor's information are stored in Database. Along these patients can utilize the database servers to inquiry the specialist data and pick the correct specialist for analysis. The patients can also input their daily life information, health reports, and infection records into the database making it simple for the specialist to give diagnosis. Aside from all these the clients can counsel specialist ongoing through voice, video and text.

The issue with this design is that there is no support for privacy and security of client information, QoS support for time sensitive or critical, data and mobility of the user. All these parameters are critical for the timely and accurate response in case of emergencies [9].

\section{COMPARISON OF THE ARCHITECTURES}

In this section, describes the outlook of the different IoT architectures (Fig: 1, Fig: 2, Fig: 3, Fig: 4) and tells about each architecture comparison. For scope of analysis the author consider the high-level attributes that describe goals and the status of the research the quality assessment focuses on the specialized parts of technologies. The individual things are portrayed in the following. In order to have the capacity to analyze and compare the architectures, at least their high-level descriptions should be available. Not all of them are considered in detail with the parameters. However, there would be already provided architectures in detailed. While Fig 1: lacks this detailed description, for the others listed here these descriptions are available in Fig1: seems to be more focused on mobile patients where patients are in go with technologies. Data captured here is not secured and can be hijacked, where as in other architecture there is a separate mechanism for security. RFID's are used in Fig 2: RFID are too expensive and too limited. All the health care units cannot afford RFID. Usage of RFID is limited by its range only nearest readers can read the data

Cloudlet is used in Fig 3: Cloudlets are limited resource computing and storage platform that eliminates the need to outsource resource intensive tasks to the enterprise cloud. 
Cloudlet computing has been introduced as a potential solution to deliver low latency to time critical tasks. Zigbee in Fig: 3 and Fig 4: facilitates the low power communication protocols, as the communication can account a significant part of the power consumption in sensing devices to support communication between low power devices that is operate in personal operating space (POS) of approximately $10 \mathrm{~m}$.
Whereas ZigBee over IEEE 802.15.4 is commonly used in low rate WPANs (LR-WPANs) so ZigBee provides reliable mesh networking with extended battery life. .The research study mainly concentrate on the attributes of IoT architectures in detail. The discoveries are introduced in Table 1 as given below.

Table 1. Comparison of Technologies

\begin{tabular}{|c|c|c|c|c|c|c|c|c|}
\hline Architectures & \multicolumn{2}{|c|}{ Technologies Used } & \multicolumn{3}{c|}{ Services Provided } & \multicolumn{2}{c|}{ IoT Interoperability } \\
\hline & RFID & WSN & Mobile & $\begin{array}{l}\text { Patient } \\
\text { Tracking }\end{array}$ & $\begin{array}{l}\text { Remote } \\
\text { Patient } \\
\text { Monitoring }\end{array}$ & $\begin{array}{c}\text { Alert } \\
\text { Notification }\end{array}$ & 6LoWPAN & $\begin{array}{l}\text { Bluetooth } \\
\text { /Zigbee }\end{array}$ \\
\hline Fig:1 & NO & YES & YES & NO & YES & YES & YES & NO \\
\hline Fig:2 & YES & YES & NO & YES & NO & NO & NO & NO \\
\hline Fig:3 & NO & YES & YES & YES & NO & YES & NO & YES \\
\hline Fig:4 & NO & YES & NO & YES & NO & NO & NO & YES \\
\hline
\end{tabular}

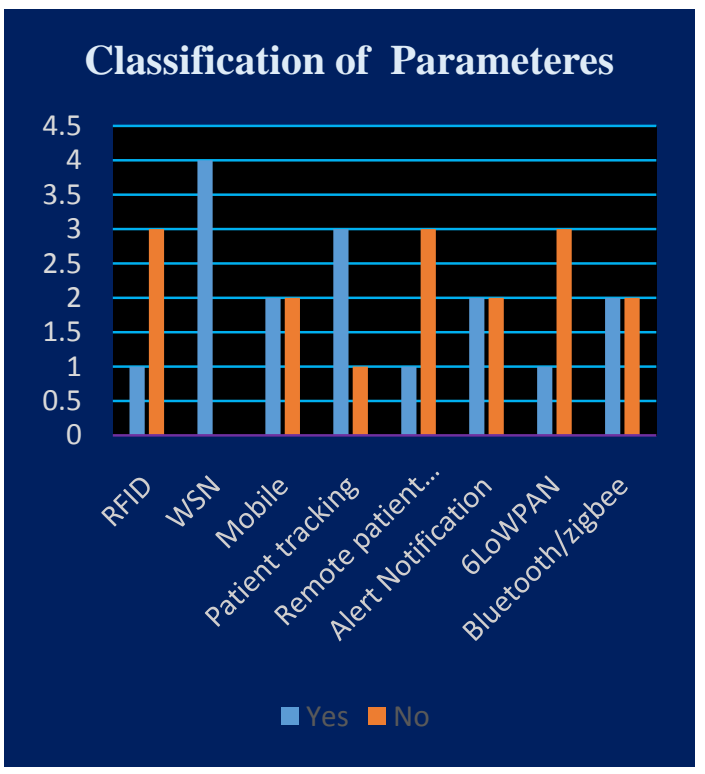

Fig 5: Comparison of different technologies with respect to architectures

The Fig 5: tells how frequently the technologies have been used in each architecture. In the graph different technologies are in the $\mathrm{x}$-axis and number of instances in y-axis. Each comparison plots in the graph with yes or no values and tells which technology has more efficient or frequently used in the hospital system. So this can be useful for doctors to decide which technology can be implementing in their hospitals.

\section{CONCLUSION AND FUTURE SCOPE}

Due to advancement in usage of Internet this work is targeted to implement the internet technology to communicate with systems for better health. Internet of things is expected to rule the world in various fields but more benefit would be in the field of healthcare. Hence present work is to compare the above 4 architectures out of which we conclude saying that Fig: 3 is more reliable architecture which uses advanced technologies like Zigbee, cloudlet and data analytics and visualization. These techniques ease the analyses of the gathered data and facilitate the clinician to make the appropriate decision. Usage of Zigbee and cloudlet lowers the design cost.

The future work IoT Healthcare can be concentrated on the rural to develop the hospital facilities because many people ignore the health issues due to poverty. The major deaths are due to late detection of treatments. Introducing IoT in rural areas will be helpful for the people to take immediate treatments. If the people are not aware of the existing technologies. The caregivers can educate them in advance to operate the technologies and this will be helpful for the patients. The study of the research is to develop an enhanced architecture which would focus on bringing IoT in health centers of rural areas with a reasonable cost.

\section{REFERENCES}

[1] Nomusa Dlodlo, Thato Foko, Promise Mvelase, and Sizakele Mathaba, CSIR Meraka Institute, Pretoria, South Africa," The State of Affairs in Internet of Things Research"

[2] 802.15.4-2003, IEEE Standard, "Wireless medium access control and physical layer specifications for lowrate wireless personal area networks", May 2003.

[3] Jaroslav Kadlec*, Radek Kuchta, Radovan Novotný and Ondřej Čožík," RFID Modular System for the Internet of Things (IoT) 
[4] E.Serafim, and S.Motoyama "A Network structure for Medical Assistance in Rural and Urban Areas using IOT Technology"

[5] Vijayakannan Sermakani,Robert Bosch Engineering and Business Ltd, "Transforming Healthcare through Internet of Things"

[6] islam1, (member, ieee), daehan kwak2, md. humaun kabir1,mahmud hossain3, and kyung-sup kwak1, (member, ieee), "the internet of things in healthcare: a comprehension survey"

[7] Byung Mun Lee, Dynamic Data Binding Protocol between IoT Medical Device and IoT Medical Service for Mobile Healthcare", International Journal of Smart Home Vol. 9, No. 6 (2015), pp. 141-150

[8] Moeen Hassanalieragh*, Alex Page*, Tolga Soyata*, Gaurav Sharma*, Mehmet Aktas\%, Gonzalo Mateos*Burak Kantarciff, Silvana Andreescu $\S$, "Health Monitoring and Management UsingInternet-of-Things (IoT) Sensing with Cloud-based Processing: Opportunities and Challenges"

[9] Tuan Nguyen Gia, Nanda Kumar Thanigaivelan, AmirMohammad Rahmani, Tomi Westerlund, Pasi Liljeberg, and Hannu Tenhunen ," Health Monitoring and Management UsingInternet-of-Things (IoT) Sensing with Cloud-based Processing: Opportunities and Challenges"

[10] RaviTejaGuthikonda,SaiSrikar,Chitta,Shraddha Tekawade, Tripti Attavar,"COMPARATIVE ANALYSIS OF IoT ARCHITECTURES",TLEN 5710 Capstone April 25, 2014

[11] 802.15.4-2003, IEEE Standard, "Wireless medium access control and physical layer specifications for lowrate wireless personal area networks", May 2003.

[12] 802.15.4-2003, IEEE Standard, "Wireless medium access control and physical layer specifications for lowrate wireless personal area networks", May 2003.Spector, A. Z. 1989. Achieving application requirements. In Distributed Systems, S. Mullender

[13] J. Holler, V. Tsiatsis, C. Mulligan, S. Karnouskos, S. Avesand, and D. Boyle, From Machine-to-Machine to the Internet of Things: Introduction to a New Age of Intelligence. Amsterdam, the Netherlands: Elsevier, 2014.

[14] http://www.zigbee.org/internet-of-things-healthcarezigbee-and-senior-lifestyle-systems/

[15] http://www.ti.com/lsds/ti/wirelessconnectivity/6lowpan/overview. 